\title{
Process Capability Indices as Fuzzy Numbers
}

\author{
Abbas Parchami, Mashaallah Mashinchi, Ali Reza Yavari, \\ and Hamid Reza Maleki \\ Shahid Bahonar University, Kerman, Iran
}

\begin{abstract}
Most of the traditional methods for assessing the capability of manufacturing processes are dealing with crisp quality. In this paper we discuss the fuzzy quality and introduce fuzzy process capability indices, where instead of precise quality we have two membership functions for specification limits. These indices are necessary when the specification limits are fuzzy and they are helpful for comparing manufacturing processes with fuzzy specification limits. Some interesting relations among the introduced indices are obtained. Numerical examples are given to clarify the method.
\end{abstract}

\begin{abstract}
Die meisten traditionellen Methoden zur Beurteilung der Qualität eines Fertigungsprozesses haben mit scharfer Qualität zu tun. In diesem Aufsatz diskutieren wir die unscharfe Qualität und führen unscharfe Indizes zur Beurteilung der Prozessleistung ein. Dazu haben wir anstatt präziser Qualität zwei Mitgliedschaftsfunktionen für die Spezifikationsgrenzen. Diese Indizes sind notwendig falls die Grenzen unscharf sind und sie erlauben den Vergleich von Fertigungsprozessen mittels unscharfer Spezifikationsgrenzen. Einige interessante Beziehungen für diese Indizes werden hergeleitet. Numerische Beispiele sind angegeben, um die Methode zu verdeutlichen.
\end{abstract}

Keywords: Fuzzy Quality Standard, Fuzzy Process Capability Index, Triangular Fuzzy Number, Fuzzy Ranking.

\section{Introduction}

The process capability compares the output of a process to the specification limits (SLs) by using capability indices. Frequently, this comparison is made by forming the ratio of the width between the process SLs to the width of the natural tolerance limits which is measured by 6 process standard deviation units. This method leads to make a statement about how well the process meets specifications (see Montgomery, 2001). A process is said to be capable if with high probability the real valued quality characteristic of the produced items lies between a lower and upper specification limit (see Kotz and Johnson, 2002). There are several statistics such as $C_{p}, C_{p k}, C_{p m}$, and so on, which are used to estimate the capability of a manufacturing process. In most cases a normal distribution and large sample sizes are assumed for the data (see Kotz and Johnson, 2002, and Kotz, 1993).

After the inception of the notion of fuzzy sets by Zadeh (1965) there are efforts by many authors, such as Viertl (1996) as well as Zadeh himself, to apply this notion in statistics (see Taheri, 2003, for such trends).

In some cases SLs are not precise numbers and they are expressed in fuzzy terms, so that the classical capability indices could not be applied. For such cases Yongting (1996) 
introduced a process capability index $C_{p}$ as a real number and it was used by SadeghpourGildeh (2003). Lee (2001) investigated a process capability index, $C_{p k}$, as a fuzzy set.

In this paper we introduce new process capability indices as triangular fuzzy numbers, where the engineering specification limits are fuzzy triangular numbers as well. The organization of this paper is as follows. In Section 2, some preliminaries are discussed. In Section 3, traditional process capability indices are reviewed. In Section 4 a fuzzy process is considered and then a new fuzzy process capability index $\tilde{C}_{p}$ as a fuzzy set is defined which seems to be more realistic than a real number used by Yongting (1996) and Sadeghpour-Gildeh (2003). Then more new fuzzy indices $\tilde{C}_{p k}, \tilde{C}_{p m}, \tilde{C}_{p m k}$ and $\tilde{C}_{p}(u, v)$ are introduced. In Section 5, the relation between the introduced fuzzy process capabilities indices are studied. In Section 6, a method based on a binary relation is presented which is used for the comparison of fuzzy processes. This method is clarified by two examples and the final section is the conclusion part.

\section{Preliminaries}

Let $\mathbb{R}$ be the set of real numbers. Let

$$
\begin{aligned}
F(\mathbb{R}) & =\{A \mid A: \mathbb{R} \rightarrow[0,1], A \text { is a continuous function }\}, \\
F_{T}(\mathbb{R}) & =\left\{T_{a, b, c} \mid a, b, c \in \mathbb{R}, a \leq b \leq c\right\}
\end{aligned}
$$

where

$$
T_{a, b, c}(x)= \begin{cases}(x-a) /(b-a), & \text { if } a \leq x<b \\ (c-x) /(c-b), & \text { if } b \leq x<c \\ 0, & \text { elsewhere }\end{cases}
$$

Any $A \in F(\mathbb{R})$ is called a fuzzy quantity on $\mathbb{R}$ and any $T_{a, b, c} \in F_{T}(\mathbb{R})$ is called a fuzzy triangular number, which we sometimes write as $T(a, b, c)$. We assume $T(a, a, a)$ be $I_{\{a\}}$, the indicator function of $a$. In this case, for simplicity, we write $T(a, a, a)=a$.

Definition 2.1. Let $A \in F(\mathbb{R})$, then

a) $A$ is called normal, if and only if there exist some $x \in \mathbb{R}$ such that $A(x)=1$.

b) $A$ is called convex, if and only if

$$
A(\lambda x+(1-\lambda) y) \geq(A(x) \wedge A(y)), \quad \forall x, y \in \mathbb{R}, \quad \forall \lambda \in[0,1],
$$

where the symbol $\wedge$ denotes the minimum operator.

c) The support of $A$ is the crisp set given by $\operatorname{Supp}(A)=\{x \mid A(x)>0\}$.

d) The $\alpha$-cut set of $A$ is the crisp set given by $A_{\alpha}=\{x \mid A(x) \geq \alpha\}$, for any $\alpha \in[0,1]$.

The following definition could be given by using the extension principle (see Nguyen and Walker, 2000).

Definition 2.2. Let $T(a, b, c), T\left(a^{\prime}, b^{\prime}, c^{\prime}\right) \in F_{T}(\mathbb{R}), k \in \mathbb{R}, k>0$ and $a \geq c^{\prime}$. Define the operations $\ominus$ and $\oslash$ on $F_{T}(\mathbb{R})$ as

$$
T(a, b, c) \ominus T\left(a^{\prime}, b^{\prime}, c^{\prime}\right)=T\left(a-c^{\prime}, b-b^{\prime}, c-a^{\prime}\right),
$$


called the width between $T(a, b, c)$ and $T^{\prime}\left(a^{\prime}, b^{\prime}, c^{\prime}\right)$, and

$$
T(a, b, c) \oslash k=T(a / k, b / k, c / k),
$$

called the division of $T(a, b, c)$ by $k$.

\section{Traditional Process Capability Indices}

A process capability index (PCI) is a real number as a summary that compares the behavior of a product or process characteristic to engineering specifications. This measure is also called performance index. Several PCIs are introduced in the literature such as $C_{p}, C_{p k}, C_{p m}$, and so on (see Kotz and Johnson, 2002, Kotz, 1993, Pearn et al., 1992). For convenience, we will denote the upper and lower specification limits by $U$ and $L$, respectively, rather than the more customary USL and LSL notations. When univariate measurements are concerned, we will denote the corresponding random variate by $X$. The expected value and standard deviation of $X$ will be denoted by $\mu$ and $\sigma$, respectively. We will limit ourselves to the situation where $\mu$ is in the specification interval, i.e. $L \leq \mu \leq U$, and we assume that the measured characteristic should have a normal distribution (at least, approximately), although it is difficult to see why a good industrial process must result in a normal distribution for every measured characteristic.

The commonly recognized PCIs are

$$
C_{p}=\frac{U-L}{6 \sigma}=\frac{w}{6 \sigma}
$$

where $w=U-L$. This $C_{p}$ is used when $\mu=M$, where $M=(U+L) / 2$.

$$
C_{p k}=\frac{w-2|\mu-M|}{6 \sigma}=\frac{\min \{U-\mu, \mu-L\}}{3 \sigma},
$$

and

$$
C_{p m}=\frac{w}{6 \sqrt{\sigma^{2}+(\mu-T)^{2}}}=\frac{w}{6 \sqrt{\mathrm{E}\left[(X-T)^{2}\right]}},
$$

where $T$ is the target value and $E[$.$] denotes the expected value. There is also the hybrid$ index

$$
C_{p m k}=\frac{w-2|\mu-M|}{6 \sqrt{\sigma^{2}+(\mu-T)^{2}}}=\frac{w-2|\mu-M|}{6 \sqrt{\mathrm{E}\left[(X-T)^{2}\right]}} .
$$

Usually, $T=M$. If $T \neq M$ the situation is sometimes described as "asymmetric tolerances" (see Boyles, 1994, Vannman, 1997, and Vannman, 1998). Introduction of $C_{p}$ is ascribed to Juran (1974), that of $C_{p k}$ to Kane (1986), that of $C_{p m}$ for the most part to Hsiang and Taguchi (1985), and $C_{p m k}$ to Pearn et al. (1992).

Clearly $C_{p} \geq C_{p k} \geq C_{p m k}$ and $C_{p} \geq C_{p m} \geq C_{p m k}$. Some more relations between PCIs could be realized. From (3) and (4), we have

$$
C_{p k}=C_{p}-\frac{1}{3}\left|\frac{\mu-M}{\sigma}\right|
$$


and from (3) and (5) we have

$$
C_{p m}=\frac{C_{p}}{\sqrt{1+\left(\frac{\mu-T}{\sigma}\right)^{2}}} .
$$

From (4) and (6), we have

$$
C_{p m k}=\frac{C_{p k}}{\sqrt{1+\left(\frac{\mu-T}{\sigma}\right)^{2}}} .
$$

A further interesting relation is

$$
C_{p m k}=\frac{C_{p m} C_{p k}}{C_{p}}
$$

An enlightening view of relations among the PCIs can be obtained from studies of the "superstructure PCIs" introduced by Vannman (1995) as

$$
C_{p}(u, v)=\frac{w-2 u|\mu-M|}{6 \sqrt{\sigma^{2}+v(\mu-T)^{2}}}, \quad u, v \geq 0 .
$$

The four PCIs introduced in (3)-(6) are special case of $C_{p}(u, v)$. Indeed

$$
C_{p}=C_{p}(0,0) ; \quad C_{p k}=C_{p}(1,0) ; \quad C_{p m}=C_{p}(0,1), \quad \text { and } C_{p m k}=C_{p}(1,1) .
$$

See more details about of this section in Kotz and Johnson (2002).

\section{Fuzzy Process Capability Indices}

As explained in Section 1, the $C_{p}$ index based on fuzzy SLs introduced as a real number by Yongting (1996) and was used by other authors. But it would be more realistic to have a $C_{p}$ which is also fuzzy, since a fuzzy process capability index would include much more information than a precise number, when we have fuzzy specification limits. For this reason, we improve Yongting's PCI and introduce $\tilde{C}_{p}$ as a fuzzy capability index. It is natural to use fuzzy numbers such as $U\left(a_{u}, b_{u}, c_{u}\right)=T\left(a_{u}, b_{u}, c_{u}\right) \in F_{T}(\mathbb{R})$ and $L\left(a_{l}, b_{l}, c_{l}\right)=T\left(a_{l}, b_{l}, c_{l}\right) \in F_{T}(\mathbb{R})$ for the upper and lower engineering specification limits, if process specification limits are fuzzy rather than real numbers.

Definition 4.1. A process with fuzzy specification limits, which we call a fuzzy process for short, is one which approximately satisfies the normal distribution condition and its specification limits are fuzzy.

Definition 4.2. Suppose we have a fuzzy process with fixed $\sigma$, for which the upper and lower specification limits are the fuzzy sets $U\left(a_{u}, b_{u}, c_{u}\right), L\left(a_{l}, b_{l}, c_{l}\right) \in F_{T}(\mathbb{R})$, where $a_{u} \geq c_{l}$. Then

a) The width between fuzzy process specification limits is a triangular fuzzy number $\tilde{w}_{S L} \in F_{T}(\mathbb{R})$, defined by

$$
\tilde{w}_{S L}=U\left(a_{u}, b_{u}, c_{u}\right) \ominus L\left(a_{l}, b_{l}, c_{l}\right) .
$$


b) The fuzzy process capability index is a triangular fuzzy number, $\tilde{C}_{p} \in F_{T}(\mathbb{R})$, defined by

$$
\tilde{C}_{p}=\tilde{w}_{S L} \oslash 6 \sigma
$$

By (1) and (2) we obtain

$$
\tilde{C}_{p}=T\left(\frac{a_{u}-c_{l}}{6 \sigma}, \frac{b_{u}-b_{l}}{6 \sigma}, \frac{c_{u}-a_{l}}{6 \sigma}\right)
$$

Note that $\tilde{C}_{p}$ is useful when $\mu=m$, where $m=\left(b_{u}+b_{l}\right) / 2$. In the following we give an example to clear the idea of $\tilde{C}_{p}$.

Example 4.1. For a special product suppose that the specification limits are considered to be "approximately 4" and "approximately 8" which are characterized by $L(2,4,6) \in$ $F_{T}(\mathbb{R})$ and $U(7,8,9) \in F_{T}(\mathbb{R})$, respectively (see Figure 1 ). Assume that the process mean $\mu$ is 6 and the estimated process standard deviation is $2 / 3$.

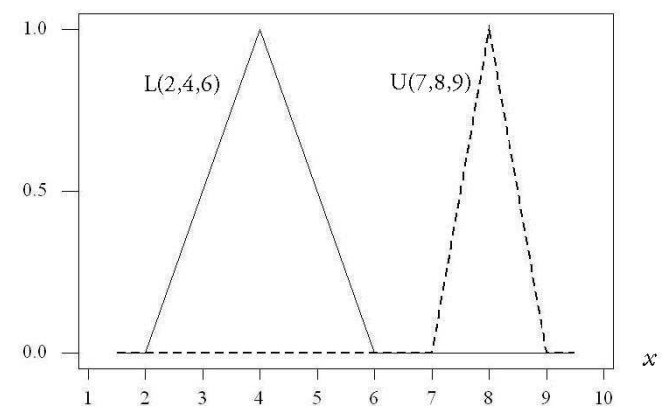

Figure 1: The membership function of fuzzy process specification limits in Example 4.1.

By Definition 4.2 we can compute the width between process SLs as $\tilde{w}_{S L}=T(1,4,7)$. Therefore, the estimation of $\tilde{C}_{p}$ is $\hat{\tilde{C}}_{p}=T(1 / 4,1,7 / 4)$. Hence, $\hat{\tilde{C}}_{p}$ is "approximately one", as shown in Figure 2.

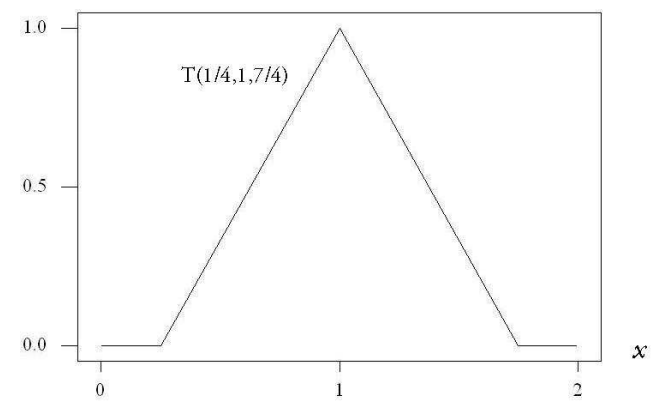

Figure 2: The membership function of fuzzy process capability index in Example 4.1

Definition 4.3. Let $U\left(a_{u}, b_{u}, c_{u}\right), L\left(a_{l}, b_{l}, c_{l}\right) \in F_{T}(\mathbb{R})$ be the upper and lower engineering fuzzy specification limits, where $a_{u} \geq c_{l}$. We introduce the following new fuzzy PCIs, 
by Definition 4.2 and the inspiration of the relations (4)-(6), as

$$
\begin{gathered}
\tilde{C}_{p k}=T\left(\frac{a_{u}-c_{l}-2|\mu-m|}{6 \sigma}, \frac{b_{u}-b_{l}-2|\mu-m|}{6 \sigma}, \frac{c_{u}-a_{l}-2|\mu-m|}{6 \sigma}\right), \\
\tilde{C}_{p m}=T\left(\frac{a_{u}-c_{l}}{6 \sqrt{\sigma^{2}+(\mu-t)^{2}}}, \frac{b_{u}-b_{l}}{6 \sqrt{\sigma^{2}+(\mu-t)^{2}}}, \frac{c_{u}-a_{l}}{6 \sqrt{\sigma^{2}+(\mu-t)^{2}}}\right) \\
\tilde{C}_{p m k}=T\left(\frac{a_{u}-c_{l}-2|\mu-m|}{6 \sqrt{\sigma^{2}+(\mu-t)^{2}}}, \frac{b_{u}-b_{l}-2|\mu-m|}{6 \sqrt{\sigma^{2}+(\mu-t)^{2}}}, \frac{c_{u}-a_{l}-2|\mu-m|}{6 \sqrt{\sigma^{2}+(\mu-t)^{2}}}\right),
\end{gathered}
$$

where $m=\left(b_{u}+b_{l}\right) / 2$ and $t$ is the target value.

The "superstructure fuzzy PCI" is defined as

$$
\tilde{C}_{p}(u, v)=T\left(\frac{a_{u}-c_{l}-2 u|\mu-m|}{6 \sqrt{\sigma^{2}+v(\mu-t)^{2}}}, \frac{b_{u}-b_{l}-2 u|\mu-m|}{6 \sqrt{\sigma^{2}+v(\mu-t)^{2}}}, \frac{c_{u}-a_{l}-2 u|\mu-m|}{6 \sqrt{\sigma^{2}+v(\mu-t)^{2}}}\right)
$$

for $u, v \geq 0$.

\section{Remark 4.1.}

i) The process capability indices defined by (3)-(6) could be expressed by the fuzzy process capability indices as

$$
\begin{aligned}
C_{p} & =\tilde{C}_{p}=T\left(C_{p}, C_{p}, C_{p}\right), \\
C_{p k} & =\tilde{C}_{p k}=T\left(C_{p k}, C_{p k}, C_{p k}\right), \\
C_{p m} & =\tilde{C}_{p m}=T\left(C_{p m}, C_{p m}, C_{p m}\right), \\
C_{p m k} & =\tilde{C}_{p m k}=T\left(C_{p m k}, C_{p m k}, C_{p m k}\right) .
\end{aligned}
$$

ii) When the process specification limits $U\left(a_{u}, b_{u}, c_{u}\right)$ and $L\left(a_{l}, b_{l}, c_{l}\right)$ are precise numbers, i.e. $a_{u}=b_{u}=c_{u}$ and $a_{l}=b_{l}=c_{l}$, then all the introduced fuzzy PCIs are precise numbers and they coincide with the traditional PCIs.

\section{Relationships between Fuzzy Process Capability Indices}

Here we give some relations fuzzy process capability indices introduced in Section 4.

Theorem 5.1. In a fuzzy process assume $a_{u} \geq c_{l}$. Then there are the following relations between fuzzy PCIs introduced in (14)-(17)

$$
\begin{aligned}
\tilde{C}_{p k}(x) & =\tilde{C}_{p}(x+|\mu-m| / 3 \sigma), \quad \text { where } m=\left(b_{u}+b_{l}\right) / 2, \\
\tilde{C}_{p m}(x) & =\tilde{C}_{p}\left(x \sqrt{1+(\mu-t)^{2} / \sigma^{2}}\right), \\
\tilde{C}_{p m k}(x) & =\tilde{C}_{p k}\left(x \sqrt{1+(\mu-t)^{2} / \sigma^{2}}\right) .
\end{aligned}
$$

Proof. Let $k=|\mu-m| / 3 \sigma$. Then by some calculations we have

$$
\tilde{C}_{p}(x+k)= \begin{cases}\frac{c_{l}-a_{u}+6 \sigma(x+k)}{b_{u}+c_{l}-b_{l}-a_{u}}, & \text { if }\left(a_{u}-c_{l}\right) / 6 \sigma \leq x+k<\left(b_{u}-b_{l}\right) / 6 \sigma, \\ \frac{c_{u}-a_{l}-6 \sigma(x+k)}{c_{u}+b_{l}-a_{l}-b_{u}}, & \text { if }\left(b_{u}-b_{l}\right) / 6 \sigma \leq x+k<\left(c_{u}-a_{l}\right) / 6 \sigma, \\ 0, & \text { elsewhere }\end{cases}
$$




$$
\tilde{C}_{p k}(x)= \begin{cases}\frac{c_{l}-a_{u}+6 \sigma(x+k)}{b_{u}+c_{l}-b_{l}-a_{u}}, & \text { if }\left(a_{u}-c_{l}\right) / 6 \sigma-k \leq x<\left(b_{u}-b_{l}\right) / 6 \sigma-k, \\ \frac{c_{u}-a_{l}-6 \sigma(x+k)}{c_{u}+b_{l}-a_{l}-b_{u}}, & \text { if }\left(b_{u}-b_{l}\right) / 6 \sigma-k \leq x<\left(c_{u}-a_{l}\right) / 6 \sigma-k, \\ 0, & \text { elsewhere. }\end{cases}
$$

Therefore, (19) is proved. Similarly we can prove (20) and (21).

Theorem 5.2. The four fuzzy PCIs introduced in (14)-(17) are special cases of $\tilde{C}_{p}(u, v)$. Indeed

$$
\tilde{C}_{p}=\tilde{C}_{p}(0,0), \quad \tilde{C}_{p k}=\tilde{C}_{p}(1,0), \quad \tilde{C}_{p m}=\tilde{C}_{p}(0,1), \quad \text { and } \tilde{C}_{p m k}=\tilde{C}_{p}(1,1) .
$$

Proof. It is obvious by using (14)-(18).

A further interesting identity among PCIs is stated in the following theorem. First we give some important lemmas.

Definition 5.1. Let $\circ: \mathbb{R} \times \mathbb{R} \rightarrow \mathbb{R}$ be an operation and $C, D \subseteq \mathbb{R}$. Define

$$
C \circ D=\{x \circ y \mid x \in C, y \in D\}
$$

Remark 5.1. One can check that if $\circ$ is the usual multiplication "." on $\mathbb{R}$ and $[a, b],[c, d]$ are real intervals in $\mathbb{R}$, where $a, b, c, d \geq 0$, then

$$
[a, b] \cdot[c, d]=[a c, b d] .
$$

See also Kaufmann and Gupta (1988) for interval multiplications.

Lemma 5.1. If $A$ and $B$ are in $F(\mathbb{R})$ with bounded support, all $\alpha$-cuts are closed, and $\circ$ is a continuous binary operation on $\mathbb{R}$, then $(A \circ B)_{\alpha}=A_{\alpha} \circ B_{\alpha}$, for all $\alpha \in[0,1]$, where $(A \circ B)(x)=\sup _{x=y \circ z} \min (A(y), B(z))$.

Proof. See Corollary 3.1.10 of Nguyen and Walker (2000).

Lemma 5.2. Let $A, B \in F(\mathbb{R})$. Then $A=B$ if and only if $A_{\alpha}=B_{\alpha}$ for all $\alpha \in[0,1]$. Proof. See Theorem 2.5.2 of Nguyen and Walker (2000).

Now the following theorem can be proved.

Theorem 5.3. Let in a fuzzy process $a_{u} \geq c_{l}$ and $\tilde{C}_{p}, \tilde{C}_{p k}, \tilde{C}_{p m}$ and $\tilde{C}_{p m k}$ be defined by (14)-(17), respectively. Then $\tilde{C}_{p} \tilde{C}_{p m k}=\tilde{\widetilde{C}}_{p m} \tilde{C}_{p k}$.

Proof. Let $T(a, b, c) \in F_{T}(\mathbb{R})$, then

$$
(T(a, b, c))_{0}=\mathbb{R}
$$

and

$$
(T(a, b, c))_{\alpha}=[a+\alpha(b-a), c+\alpha(b-c)], \quad \forall \alpha \in(0,1] .
$$

Note that $\tilde{C}_{p}, \tilde{C}_{p k}, \tilde{C}_{p m}, \tilde{C}_{p m k} \in F_{T}(\mathbb{R})$, hence we can calculate their $\alpha$-cuts from (24) as

$$
\left(\tilde{C}_{p}\right)_{\alpha}=\left[\frac{a_{u}-c_{l}}{6 \sigma}+\alpha \frac{b_{u}-b_{l}-a_{u}+c_{l}}{6 \sigma}, \frac{c_{u}-a_{l}}{6 \sigma}+\alpha \frac{b_{u}-b_{l}-c_{u}+a_{l}}{6 \sigma}\right],
$$




$$
\begin{aligned}
\left(\tilde{C}_{p k}\right)_{\alpha}= & {\left[\frac{a_{u}-c_{l}-2|\mu-m|}{6 \sigma}+\alpha \frac{b_{u}-b_{l}-a_{u}+c_{l}}{6 \sigma},\right.} \\
& \left.\frac{c_{u}-a_{l}-2|\mu-m|}{6 \sigma}+\alpha \frac{b_{u}-b_{l}-c_{u}+a_{l}}{6 \sigma}\right], \\
\left(\tilde{C}_{p m}\right)_{\alpha}= & {\left[\frac{a_{u}-c_{l}}{6 \sqrt{\sigma^{2}+(\mu-t)^{2}}}+\alpha \frac{b_{u}-b_{l}-a_{u}+c_{l}}{6 \sqrt{\sigma^{2}+(\mu-t)^{2}}},\right.} \\
& \left.\frac{c_{u}-a_{l}}{6 \sqrt{\sigma^{2}+(\mu-t)^{2}}}+\alpha \frac{b_{u}-b_{l}-c_{u}+a_{l}}{6 \sqrt{\sigma^{2}+(\mu-t)^{2}}}\right], \\
\left(\tilde{C}_{p m k}\right)_{\alpha}= & {\left[\frac{a_{u}-c_{l}-2|\mu-m|}{6 \sqrt{\sigma^{2}+(\mu-t)^{2}}}+\alpha \frac{b_{u}-b_{l}-a_{u}+c_{l}}{6 \sqrt{\sigma^{2}+(\mu-t)^{2}}},\right.} \\
& \left.\frac{c_{u}-a_{l}-2|\mu-m|}{6 \sqrt{\sigma^{2}+(\mu-t)^{2}}}+\alpha \frac{b_{u}-b_{l}-c_{u}+a_{l}}{6 \sqrt{\sigma^{2}+(\mu-t)^{2}}}\right] .
\end{aligned}
$$

Therefore, by Lemma 5.1, we have for any $\alpha \in[0,1]$

$$
\begin{aligned}
\left(\tilde{C}_{p}\right)_{\alpha} \cdot\left(\tilde{C}_{p m k}\right)_{\alpha}= & {\left[\frac{\left(a_{u}-c_{l}\right)\left(a_{u}-c_{l}-2|\mu-m|\right)}{36 \sigma \sqrt{\sigma^{2}+(\mu-t)^{2}}}+\frac{\alpha\left(a_{u}-c_{l}\right)\left(b_{u}-b_{l}-a_{u}+c_{l}\right)}{36 \sigma \sqrt{\sigma^{2}+(\mu-t)^{2}}}\right.} \\
& +\frac{\alpha\left(b_{u}-b_{l}-a_{u}+c_{l}\right)\left(a_{u}-c_{l}-2|\mu-m|\right)}{36 \sigma \sqrt{\sigma^{2}+(\mu-t)^{2}}}+\frac{\alpha^{2}\left(b_{u}-b_{l}-a_{u}+c_{l}\right)^{2}}{36 \sigma \sqrt{\sigma^{2}+(\mu-t)^{2}}} \\
& \frac{\left(c_{u}-a_{l}\right)\left(c_{u}-a_{l}-2|\mu-m|\right)}{36 \sigma \sqrt{\sigma^{2}+(\mu-t)^{2}}}+\frac{\alpha\left(c_{u}-a_{l}\right)\left(b_{u}-b_{l}-c_{u}+a_{l}\right)}{36 \sigma \sqrt{\sigma^{2}+(\mu-t)^{2}}} \\
& \left.+\frac{\alpha\left(b_{u}-b_{l}-c_{u}+a_{l}\right)\left(c_{u}-a_{l}-2|\mu-m|\right)}{36 \sigma \sqrt{\sigma^{2}+(\mu-t)^{2}}}+\frac{\alpha^{2}\left(b_{u}-b_{l}-c_{u}+a_{l}\right)^{2}}{36 \sigma \sqrt{\sigma^{2}+(\mu-t)^{2}}}\right] \\
= & \left(\tilde{C}_{p m}\right)_{\alpha} \cdot\left(\tilde{C}_{p k}\right)_{\alpha} \cdot
\end{aligned}
$$

Note that the supports of all the fuzzy PCIs, introduced by (14)-(17) are bounded. Therefore, by Lemma 5.1 and the fact that multiplication on $\mathbb{R}$ is continuous, we have

$$
\left(\tilde{C}_{p} \tilde{C}_{p m k}\right)_{\alpha}=\left(\tilde{C}_{p}\right)_{\alpha} \cdot\left(\tilde{C}_{p m k}\right)_{\alpha}=\left(\tilde{C}_{p m}\right)_{\alpha} \cdot\left(\tilde{C}_{p k}\right)_{\alpha}=\left(\tilde{C}_{p m} \tilde{C}_{p k}\right)_{\alpha}
$$

for all $\alpha \in[0,1]$. Hence, by Lemma 5.2 and (25), we conclude that $\tilde{C}_{p} \tilde{C}_{p m k}=\tilde{C}_{p m} \tilde{C}_{p k}$.

\section{Comparison of Fuzzy Processes}

When we have several fuzzy processes, a criterion for comparing of two fuzzy subsets is needed. There are many ways to do this comparison, (see Wang, 2001). We use the approach in Yuan (1991), since it is a reasonable approach and it has appropriate properties such as distinguishability and robustness. For the following definitions see Yuan (1991).

Definition 6.1. A binary relation on $F(\mathbb{R})$ is a fuzzy set such as $\mu: F(\mathbb{R}) \times F(\mathbb{R}) \rightarrow[0,1]$, where $\mu(A, B)$ represents the truth level or the strength of the relation between $A$ and $B$, 
where $\mu(A, B)=1$ means that the relation between $A$ and $B$ is true or the strongest, and $\mu(A, B)=0$ means the relation is false or the weakest.

Definition 6.2. Let $C_{i}, C_{j} \in F(\mathbb{R})$ be normal and convex, as in Definition 2.1. A fuzzy relation which compares the right spread of $C_{i}$ with the left spread of $C_{j}$, defined as

$$
\Delta_{i j}=\int_{c_{i \alpha}^{+}>c_{j \alpha}^{-}}\left(c_{i \alpha}^{+}-c_{j \alpha}^{-}\right) d \alpha+\int_{c_{i \alpha}^{-}>c_{j \alpha}^{+}}\left(c_{i \alpha}^{-}-c_{j \alpha}^{+}\right) d \alpha
$$

where

$$
c_{i \alpha}^{+}=\sup \left\{x: x \in C_{i \alpha}\right\}, \quad c_{i \alpha}^{-}=\inf \left\{x: x \in C_{i \alpha}\right\},
$$

and $C_{i \alpha}$ is the $\alpha$-cut of $C_{i}, \alpha \in(0,1]$.

From a practical point of view, especially when we operate in a fuzzy environment, it is useful to introduce a criterion regarding the degree of certainty about our decision. Yuan (1991) introduced a criterion for comparing $C_{i}$ and $C_{j}$ given in the following definition.

Definition 6.3. Suppose that the assumptions of Definition 6.2 hold. Then $\mu\left(C_{i}, C_{j}\right)$ is said to be the degree of bigness of $C_{i}$ relative to $C_{j}$, where

$$
\mu\left(C_{i}, C_{j}\right)=\frac{\Delta_{i j}}{\Delta_{i j}+\Delta_{j i}} .
$$

Definition 6.4. Let $C_{i}, C_{j} \in F(\mathbb{R})$, then

i) $C_{i}$ is bigger than $C_{j}$ if and only if $\mu\left(C_{i}, C_{j}\right)>0.5$,

ii) $C_{i}$ and $C_{j}$ are equal if and only if $\mu\left(C_{i}, C_{j}\right)=0.5$.

Using the preference relation defined for each ordered pair, it is easy to rank $n$ alternatives $\left\{C_{1}, C_{2}, \ldots, C_{n}\right\}$. The procedure is as follows:

Calculate $\mu\left(C_{i}, C_{j}\right)$ for $i=1, \ldots, n, j=1, \ldots, n$, which consists of an $n \times n$ matrix. By using the fact that $\mu\left(C_{i}, C_{j}\right)=1-\mu\left(C_{j}, C_{i}\right)$, we only need to calculate $n(n-1) / 2$ membership values. Then sort $\left\{C_{1}, C_{2}, \ldots, C_{n}\right\}$ into $\left\{C_{k_{1}}, C_{k_{2}}, \ldots, C_{k_{n}}\right\}$ so that for any $i<j, \mu\left(C_{k_{i}}, C_{k_{j}}\right) \geq 0.5$. Based on the sorting we can conclude that $C_{k_{1}}$ is the most preferred choice, $C_{k_{2}}$ is the second.

Now, we use this method to compare the fuzzy PCIs of fuzzy processes in the next examples.

Example 6.1. Suppose that we want to choose one of the three fuzzy processes which is better than others, where their PCIs are obtained as follows (Figure 3)

$$
\tilde{C}_{p_{1}}(x)=T(5 / 6,1,3 / 2), \quad \tilde{C}_{p_{2}}(x)=T(2 / 3,5 / 6,1), \quad \tilde{C}_{p_{3}}(x)=T(1 / 2,7 / 6,4 / 3) .
$$

In Table 1, we have presented the results of the pairwise comparison for three fuzzy process capability indices by the aid of Maple software. For example, $\tilde{C}_{p_{1}}$ is bigger than $\tilde{C}_{p_{2}}$ with 0.93 degree of bigness and so the first fuzzy process is better than the second one. Actually we have the sorting $\left\{\tilde{C}_{p_{1}}, \tilde{C}_{p_{3}}, \tilde{C}_{p_{2}}\right\}$. Based on the sorting we can conclude 


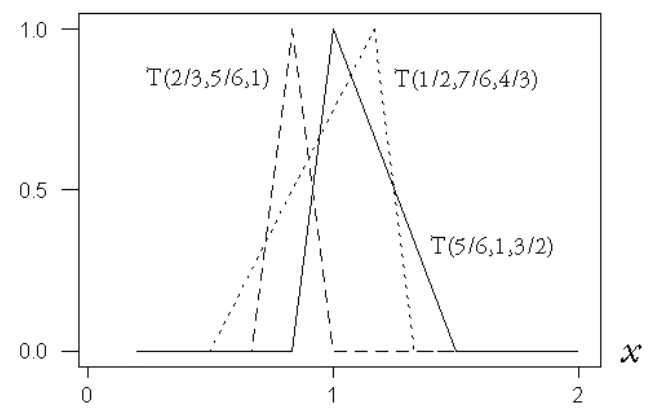

Figure 3: The membership functions of fuzzy capability indices in Example 6.1

Table 1: The results of calculations for Example 6.1

\begin{tabular}{|c|c|c|c|c|}
\hline Comparison between & $\Delta_{i j}$ & $\Delta_{j i}$ & Better fuzzy process & Degree of bigness \\
\hline$\tilde{C}_{p_{1}}$ and $\tilde{C}_{p_{2}}$ & 0.542 & 0.042 & 1 is better than 2 & 0.93 \\
\hline$\tilde{C}_{p_{1}}$ and $\tilde{C}_{p_{3}}$ & 0.429 & 0.345 & 1 is better than 3 & 0.55 \\
\hline$\tilde{C}_{p_{2}}$ and $\tilde{C}_{p_{3}}$ & 0.150 & 0.567 & 3 is better than 2 & 0.79 \\
\hline
\end{tabular}

that the first fuzzy process is the most preferred choice and the second fuzzy process is the worst choice between all three fuzzy processes.

In classical case we compare our PCIs with the number 1. In the fuzzy case we do this comparison as in the following example.

Example 6.2. In Example 6.1, we want to compare fuzzy PCIs of three fuzzy processes with "approximately one", and suppose that "approximately one" is defined by $\tilde{1}=T(5 / 6,1,7 / 6)$. In Table 2 , we have presented the results of the pairwise comparison of three fuzzy process capability indices with "approximately one" by the aid of Maple software. For example, $\tilde{C}_{p_{1}}$ is bigger than "approximately one" with 0.67 degree of bigness and so the first fuzzy process is approximately in control. Note that the decision maker can change the membership function of $\tilde{1}$.

Table 2: The results of calculations for Example 6.2

\begin{tabular}{|c|c|c|c|c|}
\hline Comparison between & $\Delta_{i j}$ & $\Delta_{j i}$ & Result of comparison & Degree of bigness \\
\hline$\tilde{C}_{p_{1}}$ and $\tilde{1}$ & 0.333 & 0.167 & $\tilde{C}_{p_{1}}$ is bigger than $\tilde{1}$ & 0.67 \\
\hline$\tilde{C}_{p_{2}}$ and $\tilde{1}$ & 0.042 & 0.375 & $\tilde{C}_{p_{2}}$ is smaller than $\tilde{1}$ & 0.10 \\
\hline$\tilde{C}_{p_{3}}$ and $\tilde{1}$ & 0.349 & 0.269 & $\tilde{C}_{p_{3}}$ is bigger than $\tilde{1}$ & 0.56 \\
\hline
\end{tabular}

\section{Conclusion}

In this paper we introduced the fuzzy process capability indices (PCIs), when the engineering specification limits (SLs) are triangular fuzzy numbers. Also several relations between them are revealed. If we define the SLs by fuzzy quantities, it is more appropriate 
to define the PCIs as fuzzy numbers. The new indices are very flexible and hence capable in manufacturing processes. A meaningful application of these new PCIs emereged when we are required to compare fuzzy processes. This is clarified by examples.

\section{Acknowledgement}

The authors would like to thank Dr. S.M. Taheri for his constructive suggestions and comments. Also, thanks to an anonymous referee for his or her comments.

\section{References}

Boyles, R. A. (1994). Process capability with asymmetric tolerances. Communications in Statistics - Simulation and Computation, 23, 613-643.

Hsiang, T. C., and Taguchi, G. (1985). Tutorial on quality control and assurance - The Taguchi methods. Joint Meetings of the American Statistical Association.

Juran, J. M. (1974). Juran's Quality Control Handbook (3rd ed.). New York: McGrawHill.

Kane, V. E. (1986). Process capability indices. Journal of Quality Technology, 18, 41-52.

Kaufmann, A., and Gupta, M. M. (1988). Fuzzy Mathematical Models in Engineering and Management Science. Amsterdam: North-Holland.

Kotz, S. (1993). Process Capability Indices. New York: Chapman and Hall.

Kotz, S., and Johnson, N. (2002). Process capability indices - a review, 1992-2000. Journal of Quality Technology, 34, 2-19.

Lee, H. T. (2001). $c_{p k}$ index estimation using fuzzy numbers. European Journal of Operational Research, 129, 683-688.

Montgomery, D. C. (2001). Introduction to statistical quality control. New York: Wiley.

Nguyen, H. T., and Walker, E. A. (2000). A First Course in Fuzzy Logic (2nd ed.). London: Chapman and Hall/CRC.

Pearn, W. L., Kotz, S., and Johnson, N. L. (1992). Distribution and inferential properties of capability indices. Journal of Quality Technology, 24, 41-52.

Sadeghpour-Gildeh, B. (2003). Comparison of $c_{p}, c_{p k}$ and $\tilde{c}_{p}$ process capability indices in the case of measurement error occurrence. In IFSA world congress (p. 563-567). Istanbul, Turkey.

Taheri, S. M. (2003). Trends in fuzzy statistics. Austrian Journal of Statistics, 32, 239257.

Vannman, K. (1995). A unified approach to the capability indices. Statistica Sinica, 5, 805-820.

Vannman, K. (1997). A general class of capability indices in the case of tolerances. Communications in Statistics - Theory and Methods, 26, 2049-2072.

Vannman, K. (1998). Families of capability indices for one-side specification limits. Statistics, 31, 43-66.

Viertl, R. (1996). Statistical Methods for Non-Precise Data. Boca Raton: CRC Press.

Wang, X. (2001). Reasonable properties for the ordering of fuzzy quantities (II). Fuzzy Sets and Systems, 118, 387-405. 
Yongting, C. (1996). Fuzzy quality and analysis on fuzzy probability. Fuzzy Sets and Systems, 83, 283-290.

Yuan, Y. (1991). Criteria for evaluating fuzzy ranking methods. Fuzzy Sets and Systems, 43, 139-157.

Zadeh, L. A. (1965). Fuzzy sets. Information and Control, 8, 338-359.

Author's addresses:

Abbas Parchami, Mashaallah Mashinchi, Ali Reza Yavari, Hamid Reza Maleki

Faculty of Mathematics and Computer Science

Shahid Bahonar University of Kerman

Kerman, Iran

Tel. \& Fax ++98 3413221080

E-mail: a_parchami@yahoo.com, mashinchi@mail.uk.ac.ir, yavari@graduate.uk.ac.ir, maleki@mail.uk.ac.ir 